\title{
Subjetividad política y psicologías sociales críticas en Latinoamérica: ideas a dos voces*
}

Political subjectivity and critical social psychologies in Latin American: Ideas to two voices

\author{
Álvaro Díaz GÓMEZ ** \\ Universidad Tecnológica de Pereira, Pereira, Colombia \\ Universidad de Manizales, Manizales, Colombia \\ FERNANDO GONZÁlez ReY \\ Centro Universitario de Brasilia, Brasil
}

Para citar este artículo: Díaz, A. (2012). Subjetividad política y psicologías sociales críticas en Latinoamérica: ideas a dos voces [Entrevista con el psicólogo cubano Dr. Fernando González Rey]. Universitas Psychologica, 11(1), 325-338.

* Entrevista realizada en el mes de octubre de 2010 en la Universidad del Valle, Cali, Colombia.

** Facultad de Bellas Artes y Humanidades. Vereda la Julita, piso 4, oficina, 401.E-mails: adiaz@utp.edu. co, gonzalez_rey49@hotmail.com. Researcher ID: Díaz, A., B-9388-2012
RESUMEN

En la presente entrevista, se desarrolla una conversación entre los psicólogos Fernando González Rey (Cuba/Brasil) y Álvaro Díaz Gómez (Colombia) sobre lo que, desde la trayectoria de vida académica de cada uno de ellos, se asume qué es la Psicología Social crítica en Latinoamérica y cómo se expresa a través de diversas opciones. Igualmente, se avanzan ideas respecto de la subjetividad en la tensión subjetividad social/subjetividad política. Palabras clave autores:

Psicología latinoamericana, psicología social crítica, subjetividad.

Palabras clave descriptores:

Fernando González Rey, entrevistas, psicología social crítica latinoamericana, subjetividad social, subjetividad política. 


\section{Algunos datos biográficos de Fernando González Rey}

Psicólogo formado en la Facultad de Psicología de la Universidad de la Habana (Cuba). Doctor en Psicología del Instituto de Psicología General y Pedagógica de Moscú. Postdoctorado en Psicología por el Instituto de Psicología de la Academia de Ciencias de la Unión Soviética.

Ha escrito, entre otros, los siguientes libros: Psicología humanista. Actualidad y desarrollo (1994), Epistemología cualitativa y subjetividad (1997), La investigación cualitativa en psicología (1999), Sujeto y subjetividad. Una aproximación Histórico-cultural (2002), O social Na psicología e A psicología social. A emergencia do sujeito (2004), Investigación cualitativa y subjetividad. Los procesos de construcción de la información (2007). Su más reciente publicación es el libro, El pensamiento de Vygotsky: contradicciones, desdoblamientos y desarrollo (2010).

Coautor de más de siete obras, ha colaborado en más de diez libros, ha publicado más de 80 artículos en revistas internacionales especializadas en español, inglés, portugués y ruso. Es miembro del Consejo Editorial de la Revista Cubana de Psicología, Psicología y Sociedad (revista de la Asociación Brasileña de Psicología Social), la revista Diversitas (Colombia), la Revista de Ciencias Humanas (Colombia), la revista Perspectivas en Psicología (Colombia).

Recibió el Premio Interamericano de Psicología (1991), la orden Carlos J. Finlay, máxima distinción del Estado cubano para investigadores científicos (1995) y, en tres ocasiones, el Premio a la Crítica, otorgado por el Instituto del Libro de Cuba.

Fue presidente de la Sociedad de Psicólogos de Cuba (1986-1999), decano de la Facultad de Psicología de la Universidad de la Habana (1985-1990) y vicerrector de la misma (1990-1995). Profesor titular de esta Universidad en la que trabajó hasta 1999. Actualmente es profesor del Centro Universitario de Brasilia, Brasil.

Estuvo en Colombia en el año 1984 y 1986 en la Facultad de Psicología de la Universidad INCCA, y en el año 2005 invitado como conferencista en el Doctorado de Salud Pública de la Universidad
Nacional de Colombia y en el Doctorado en Ciencias Sociales, Niñez y Juventud, de la Universidad de Manizales y el CINDE. Desde entonces, ha participado con frecuencia en diversas actividades académicas en este país.

\section{I.- Psicologías sociales críticas o segunda crisis de relevancia de la psicología social latinoamericana}

-Álvaro Díaz Gómez (A.D.G.): Fernando, es un placer tener esta conversación. La última vez lo habíamos hecho en la Universidad Nacional de Colombia, sede Bogotá, en el 2008. Le propongo que en esta oportunidad hablemos en torno a dos ejes temáticos: primero, nuestra apreciación sobre la situación actual de las psicologías sociales críticas en América Latina; segundo, algunas ideas sobre el tema central de nuestras reflexiones actuales: $\mathrm{La}$ subjetividad política.

-Fernando González Rey (F.G.R): De acuerdo, me parece una ruta sugestiva.

-A.D.G.: Cuando uno lee manuales sobre psicología social, generalmente encuentra un capítulo historiográfico sobre su desarrollo, enraizándolo en la tradición Europea (León, Barriga \& Gómez, 1998). Pero, si esto se hace desde textos escritos en Latinoamérica (Correa, 2003), se hace énfasis en lo que se empieza a denominar en la década del 70, como psicología social latinoamericana. Encontrando, a partir de la década del 2000 una diáspora conocida como psicología social crítica (González Rey, 2008; Montero, 2010). En ese contexto, una hipótesis que le propongo es la siguiente: la psicología social latinoamericana empieza a emerger a partir de lo que en ese momento se conoció como "la crisis de relevancia de la psicología social" y relacionada con la pregunta ipara qué le servía a la psicología latinoamericana, la psicología que provenía de Europa y de Estados Unidos? Desde allí, empieza a emerger la tendencia de la psicología social latinoamericana que no se presenta de manera unívoca, sino que se pluraliza. Al momento, estas diferentes psicologías sociales están en repliegue, 
anunciando una segunda crisis de relevancia, que se enmarcan bajo la denominación de psicologías sociales criticas, ¿cómo ve esa hipótesis?

-F.G.R.: Álvaro, me parece muy interesante la hipótesis. Cuando tú hablas de la psicología tradicional, haces una unidad entre la psicología social norteamericana y la europea, frente a la que no estoy de acuerdo, porque la primera se caracterizó por su positivismo e individualismo. Sin embargo, con Tajfel, pero particularmente con Moscovici, en la década de los años 60, aparece una psicología social que empieza a centrarse en una producción propiamente social, a través del concepto de representación social. La teoría de las representaciones sociales ha sido un campo extraordinariamente amplio que también se ha desarrollado en expresiones positivistas que banalizaron, con frecuencia, el potencial sugestivo que ese concepto posee. Pero la representación social, como la ven Moscovici o Jodelet, es una producción psíquica de carácter social, de la que se derivan muchos aspectos que han sido poco explotados, algunos de los cuales pasaron a ser criticados, en sus inicios, desde la óptica de la psicología social critica por el hecho -como lo decían los autores construccionistas- de presentar una teoría representacional de la realidad; creo que la representación social no es una entidad, si la vemos así, estamos perdidos. Creo que la representación social es una producción simbólica que nos permite generar inteligibilidad a una multiplicidad de formas de organización de lo social que pasan inadvertidas a sus protagonistas, a pesar de que los teóricos del tema no han colocado su énfasis en ese aspecto. Sin embargo, tanto las investigaciones fundadoras de Moscovici, como posteriormente las de Jodelet y Herlitch, permiten ratificar lo que afirmo. Uno de los problemas que ha tenido la teoría de las representaciones sociales es su banalización en muchas de las investigaciones científicas que la evocan, lo cual es fácil de entender por la forma en que históricamente las teorías han sido usadas en psicología. Por ejemplo, muchas de las investigaciones que sobre este tema se llevan a cabo en América Latina, se centran en la representación social del enfermero, del profesor y se queda en un nivel de descripción, de culto o de reificación a una manera pasiva de comprender la representación, con esto se ignora, incluso, algo que Moscovici dijo en la década de los 80: representaciones sociales aparecen donde Freud había colocado el inconsciente, o sea, no son producciones conscientes que se dan en la expresión directa de lo que pensamos, por lo que tenemos que buscarlas en formas ocultas de prácticas simbólicas que, de hecho, constituyen vías de inteligibilidad para un sinnúmero de procesos sociales complejos.

De todas maneras coincido contigo en esa psicología social crítica, que representó una alternativa a las formas acríticas de importación del pensamiento psicológico hegemónico en América Latina. Esa psicología social crítica, en nuestro continente, a principios de los años 80 , se caracterizó por la producción de núcleos de pensamiento distintos, que convergieron en un proceso muy interesante y que se caracterizaron por su originalidad y movilidad. Recuerdo los trabajos de todos los que estuvimos implicados en este movimiento: Martín Baró (1989), quién fue una figura central en lo que él llamo la psicología social de la liberación y quien estuvo muy comprometido con problemas de su país, del Salvador, a través de los cuales de hecho estaba fundando otra forma de pensar la psicología social; Maritza Montero (2002), quien tuvo un protagonismo muy grande en la fundamentación y visibilización de la psicología comunitaria en América Latina; los trabajos de Bernardo Jiménez (1994), de Pablo Fernando Christlieb (2004). En Brasil, la obra de Silvia Lane marcó el inicio de una psicología social crítica que mantiene un importante espacio académico y práctico hasta hoy. Si tú analizas, todos esos esfuerzos iban por rutas críticas diferentes, pero encontraron puntos de coexistencia en los que, de forma promisoria, se dieron desdoblamientos que marcaron un núcleo generador de pensamiento en la psicología social latinoamericana. Sin embargo, por razones contextuales, históricas, teóricas y otras de diferente carácter, ese núcleo se fue diluyendo, los pensadores siguieron siendo críticos cada uno por su camino, y ese legado no encontró un desarrollo consistente 
posterior. Creo que el rótulo de "psicología crítica" después fue asumido para justificar, desde lo "ideológicamente correcto", una mezcla ecléctica de investigaciones empíricas bastante limitadas, sin avanzar por caminos sólidos en el desarrollo de alternativas consistentes a las teorías dominantes. Como Martín Baró enfatizó, una psicología crítica tiene que avanzar en lo teórico, lo epistemológico y lo práctico de forma simultánea; no hay peor enemigo de la crítica que el "practicismo" disfrazado en lo "ideológicamente correcto", que es lo que ha acontecido con el carácter a-critico y cómplice del poder dominante con muchos grupos de izquierda en la región, sobre todo aquellos que han llegado al poder. Es necesario buscar nuevos caminos interdisciplinarios, retomar autores que estuvieron muy presentes en algunos de los trabajos de esa psicología crítica en sus comienzos, como Franks Fanon y Fals Borda, es necesario nutrir el pensamiento psicológico e ir haciendo nuevas corrientes al interior de nuestra psicología. A veces se usa el rótulo de psicología social crítica, para ocultar un cierto conservadurismo tradicional de categorías bastantes manidas y trilladas, con citas un poco impactantes que escapan de lo que es un núcleo teórico vivo, con capacidades generadoras múltiples.

A veces, se cae en formas de discursos políticos un poco artificiales y no tan novedosos para discutir lo que está aconteciendo políticamente en el Continente hoy. Veo hoy un cierto discurso lleno de frases y posiciones ideológicas explícitas, pero donde no percibo progreso en asuntos que fueron muy importantes en el movimiento fundador de esa psicología social crítica: la definición de problemas nuevos, el avance sobre los mismos en una discusión que se alimentaba de la diversidad y que dejaba ver nuevos caminos.

Tengo que confesarte que cuando empecé a incursionar en este movimiento de la psicología social latinoamericana con Maritza Montero, con José Miguel Salazar -un hombre de una militancia política incuestionable en América Latina-, yo venía con mis trabajos sobre personalidad desde la perspectiva crítica del Marxismo, pero todavía no había incorporado una visión problematizadora y abarcadora de lo social en su expresión más plena
(González Rey, 1994), pues en la psicología soviética, en la que me formé, la psicología social era uno de sus puntos más débiles, lo que he analizado en trabajos recientes (González Rey, 2004, 2010, 2011).

-A.D.G.: Quiero presentar un matiz frente a mi primer comentario y a la manera como usted lo interpreta: planteo una psicología social tradicional venida de Europa y Norteamérica, pero usted me responde diciendo que quizá la europea es un poco más social.

-F.G.R.: iEs totalmente más social!

-A.D.G.: Sí, pero no en la psicología tradicional que nos llegó acá, porque Moscovici es más reciente en cuanto aparece en la década del 80 y 90, pero lo que llegó antes, en el 50,60, es todavía muy individualista, por lo que se debe hacer una separación más fina y mostrar que hay otro momento de la psicología europea donde se abre la mirada, por vía de esos autores.

-F.G.R.: Concuerdo con eso. Inclusive, es una mirada que está muy lejos de haber sido bien explotada en todas sus consecuencias alternativas, porque fue interrumpida por una crítica vigorosa de los autores que se sumaban al tipo de discurso de moda en el periodo posestructuralista -las practicas discursivas- y que reificaron la práctica, por encima de todos los tejidos sociales posibles y de todas las formas de producción social diversas, que no necesariamente nos remiten a la idea de práctica discursiva.

-A.D.G.: En Colombia, pienso que por colonialismo intelectual, hay más influencia de la literatura científica norteamericana que de la europea, al menos hasta la década de los 80; ahora, con la globalización y la Internet, se ha abierto el espectro de fuentes de información, debilitando o complementando la perspectiva norteamericana, lo que conduce a otras maneras de conocer los desarrollos de la psicología social en Latinoamérica con influencias -aunque muy recientemente y esa 
es solo una vía de expresión de la psicología social europea-como las de Moscovici.

-F.G.R.: Y, sin embargo, María Auxiliadora Banchs, quien fue discípula y la primera doctora formada por Moscovici, en América Latina, trajo en los 70 la discusión sobre su pensamiento al grupo de psicología social critica, que en esos años comenzaba a desarrollarse en América Latina, defendiendo y creando opciones para integrar la representación social en este marco de reflexiones teóricas; creo que el de ella es un trabajo valioso e interesante.

-A.D.G.: Claro, era lo emergente en ese momento en cuanto quería ser núcleo generador de pensamiento, pero no lo hegemónico.

-F.G.R.: iNo!, iDe ninguna manera hegemónico! Pues como ocurre hasta ahora, los discursos de la psicología hegemónica son tremendamente positivistas y aún arrastran las categorías de actitud, de medición de actitudes, de normas separadas de contexto, como se puede apreciar en los programas académicos de las universidades latinoamericanas.

-A.D.G.: Si hacemos un desplazamiento en el recuerdo podemos evocar un texto de Munné (1982), titulado Psicologías sociales marginadas: la línea de Marx en la psicología social, donde él hace un rastreo de lo que ocurre en ese momento en Europa, su planteamiento central es que existe una serie de psicologías que no han sido reconocidas, por ejemplo, las corrientes marxistas que todavía pueden tener potencialidad, pero que no han sido desarrolladas al no ser hegemónicas.

Me parece que en América Latina hay unas psicologías sociales marginadas, por ejemplo, la psicología de la liberación, sería una de ellas. Alguna tendencia de la psicología social crítica -si se quiere radical- sería marginal, otra psicología social crítica podría, por vía del socio-construccionismo, ser una psicología social marginal; sin embargo, pienso que soplan unos nuevos vientos y que se podría hacer tanto la crítica como el complemento a esa manera de asumir el pensamiento de Moscovici en América Latina, sobre todo si se asume la representación social, para que sea complementada con la idea de imaginario social, propuesta por Castoriadis, aunque esto nos lleva por otra línea de pensamiento como es la que presenta este autor, manejando las tensiones propias de asumir dos categorías propuestas en tiempo distintos y por autores diferentes. Desde la perspectiva en la que venimos hablando, estas son influencias de Europa, pero que no marcan una nuez de psicología social latinoamericana ¿cómo podemos caracterizar esos rasgos de lo que se produce en América Latina, no en chovinismo, sino en dialogo plural?

-F.G.R.: Mira, todas estas cosas son difíciles de construir. Sin embargo, creo que toda la construcción de la psicología social comunitaria en América Latina, por ejemplo desde la producción de Maritza Montero (2003, 2004), I. Serrano $(1999,2002)$ y M. A. Tovar (1999) tiene aportes importantes. Ahora, si hablo en primera persona, el concepto de subjetividad que ayudo a impulsar es importante, pues la propongo como subjetividad social (González Rey, 1997), como una manera de traer a la discusión una idea que había sido apartada, cuando cundió una especie de alergia a todo lo que sonara a ontología, por el parentesco de lo ontológico con lo metafísico; sin embargo, nos separamos de la discusión sobre la naturaleza de lo psíquico y llegó un momento en el que reificamos en el lenguaje otros registros que no son del lenguaje y que son muy importantes para hacer una lectura de lo social. Por eso hablo de la subjetividad social. De hecho autores como Castoriadis, me influyeron.

Yo incorporo en la reflexión sobre la subjetividad social la relación recursiva imprescindible con la subjetividad individual del sujeto (González Rey, 2002), que es algo que se perdió en la psicología social. Este ha sido un tema de diálogo con los autores de las representaciones sociales, porque a veces cuando hablamos de la representación social como una producción simbólica, que delimita las posibilidades de nuestras prácticas y producciones en un contexto dado, nos olvidamos que esas representaciones tienen un alimento emocional que no está en la representación en sí, sino que está en las configuraciones subjetivas que esa representación 
toma en los sujetos y en las formas de relación de esos sujetos.

Entonces, esa representación social es portadora de una emocionalidad, cuestión esta que representó una dificultad para Moscovici, quien, en determinado momento del desarrollo de su teoría, asoció la emoción con la actitud en el concepto de representación social, lo que después supera, aunque la cuestión haya seguido en abierto hasta hoy como desafío para el desarrollo de la teoría. Sin embargo, para mí nunca ha quedado clara la presencia de esa emocionalidad como producción social que no puede prescindir de los sujetos en relación, en campos simbólicos de prácticas simbólicas. Creo que esa es una idea importante, inclusive en un libro que acaban de organizar Ángela Arruda y Mireya Lozada, donde Denise Jodelet me reconoce la importancia de haber traído el tema de la subjetividad al campo de la representación, ese es un aporte latinoamericano.

Álvaro, he sostenido durante largo tiempo una preocupación por no separar los problemas de la práctica y de los desdoblamientos de la psicología, de la construcción teórica de la misma. Creo que no nos podemos apartar de eso.

Hay otros autores con aportes como Pablo Fernández Christlieb, quien ha hecho una construcción sobre la emoción que es interesante. Están las producciones de Bernardo Jiménez (2008), trayendo conceptos desde la psicología social a la definición de las estructuras de la ciudad, en otros planos de reflexión. Hay muchos trabajos y siempre que hablo de esto me interesa dejar claro -y que quede escrito- que no se pretende abarcar el tema, ni abarcar todos sus campos significativos; en Brasil hay grupos por ejemplo el de la PUC, de Sao Pablo, que ha articulado el tema de la subjetividad al núcleo original que ellos tuvieron de una reflexión crítica que va encontrando formas distintas de expresión. Creo que se van haciendo cosas. En Colombia, los grupos de investigación en los cuales tú participas, uno desde la Universidad de Manizales-CINDE a través del Doctorado en Ciencias Sociales, Niñez y Juventud con su línea de subjetividad política (Alvarado, Ospina \& Muñoz, 2008; Botero 2007; Díaz, 2009a) y otro, desde el Grupo de Arte y Cultura, de la Universidad Tecnológica Pereira (Díaz, 2007a, 2007b, 2008), con su línea de investigación en socialización política y cultura política, han ganado voz y están sembrando un área de inteligibilidad sobre problemas que habían quedado fuera de otras construcciones teóricas.

Lo que caracteriza hoy la creación en América Latina se da por líneas que no siempre se encuentran en posibilidades de diálogo y de replanteamiento conjunto, que es lo que caracterizó la época de oro del inicio crítico, cuando nos reuníamos en Caracas con Maritza Montero, José Miguel Salazar, Bernardo Jiménez, Martín Baró, Ignacio Dobles, entre otros, participando de congresos, seminarios, cursos, proyectos escriturales y editoriales conjuntos. También están las articulaciones de Esther Wiesenfeld y Euclides Sánchez sobre la psicología comunitaria. En ese momento, había un movimiento muy bueno en América Latina. Hoy, se presentan algunos puntos en dispersión.

Se está construyendo creativamente, pero no se da el suficiente diálogo y punto de encuentro entre estas perspectivas. En mi artículo, en la Revista Interamericana de Psicología, digo que la seducción por el discurso de moda del socio-construccionismo -cuando comenzaba y que permeó los esfuerzos de la psicología social crítica- le quitó luz y voz a las iniciativas que se iban gestando en América Latina. Vino un momento de importación socioconstruccionista que no fue hegemónico, pero que sí desunió las voces críticas, hablar de prácticas discursivas entró en moda y todo lo demás fue marginal. Ahora todo va encontrando nuevos puntos de conciliación y discusión.

-A.D.G.: Fernando, a manera de síntesis de lo conversado hasta el momento, veo tres rasgos de lo que puede ser una psicología social crítica: primero, la discusión sobre los fundamentos de las metodologías mediante las cuales se investiga desde esta acepción de psicología; segundo, la posibilidad de reconocer los vínculos de interdisciplinariedad, al momento de producir conocimiento y tercero, la posibilidad de que se tengan discusiones y posturas políticas frente a contextos específicos. 
-F.G.R.: De acuerdo, esto es lo fundamental.

-A.D.G.: En Colombia, desde la red de psicología social y crítica (Díaz, 2009b; Obando, 2010), planteamos como una característica de la psicología social crítica es que esta debe ser crítica de todas las expresiones de psicología. En principio, esto puede parecer un eslogan, pero creo que hay fundamentos interesantes de trasfondo. Ahora, pensar en una psicología social crítica implica conocer la teoría de punta de esta área del conocimiento, para desde allí desarrollar un pensamiento en doble vía: un pensamiento individual, del psicólogo social crítico y un pensamiento como expresión colectiva, gremial, de comunidad académica, que necesariamente debe ser un pensamiento no solo crítico, sino autónomo...

-F.G.R.: ...Autónomo quizá no, pero especificado, porque lo que creemos autónomo siempre se configura de lo que existe, de tradiciones. Por eso digo: especificado, con capacidad de ruptura respecto de los modelos teóricos hegemónicos y de compromiso con la sociedad, no solo en el plano discursivo, sino una preocupación por articular y avanzar conceptual y prácticamente sobre problemas de la sociedad. Eso es lo que marca una psicología social crítica. Todo esto va enmarcando un corpus teórico en crecimiento, que permite nuevas producciones que le dan inteligibilidad a fenómenos que están ocultos y que están marcando de forma decisiva la realidad, eso es importante.

-A.D.G.: Podemos reconocer otros dos rasgos de esta psicología social crítica; uno, cómo pensar la temporalidad actual...

-F.G.R.: iClaro! La realidad que nos corresponde...

-A.D.G.: ...Segundo, cómo ese pensamiento se ejerce en la realidad actual -no en lo que otros nos han legado, aunque no se desconoce- lo que implica investigar en esta temporalidad, lo que es emergente.
-F.G.R.: O investigarlo, para no caer en la trampa del practicismo, y reconocer que nuestras producciones tienen la capacidad de diálogo con los problemas que tenemos. Porque a veces en un aparente problema de investigación no está lo social critico, explícito, pero está el conocimiento de formas de subjetivación al interior de una sociedad que terminan construyendo un corpus critico de las prácticas institucionales, de las prácticas familiares, que es tema de la psicología social crítica, porque no puede haber un sociologicismo discursivo que limite o estreche tipos de problemas de investigación.

Me acuerdo mucho cuando se hablaba sobre ¿cuál es la importancia social de la investigación? Y había que ofrecer un discurso sobre eso. Pienso que la importancia de la investigación social está en la calidad de lo que se construye, en su capacidad de articularse a los múltiples procesos que convergen en la sociedad (González Rey, 1999, 2007). Porque el problema es que la sociedad aparece tanto en el individuo, en la clínica, como en la organización de la escuela, como aparece en las prácticas que están implicadas en el fracaso escolar. Por donde quiera, tú tienes salidas que pueden contribuir en la constitución de un núcleo organizador muy fuerte de una psicología social critica. Pero no se descubren los temas a priori, al contrario, para poder entrar con mi contribución, tengo que trabajar lo que estoy construyendo para hacer la negociación con la visión de crítica a la sociedad y al contexto en el que me encuentro, para evitar el tipo de dogma en el cual, desde la declaración del tema, está implícita su importancia social; puedo proponer un tema con una grandilocuencia de importancia crítica y después dar resultados espurios para pensar la crítica. Esto pasa por el compromiso del investigador.

-A.D.G.: En esos términos, no se puede partir solo del compromiso con los sectores desfavorecidos de la sociedad, sino un compromiso incluso como disciplina, de donde surge la posibilidad de trabajar colectivamente con los gremios de psicólogos, porque a veces lanzamos un discurso hacia los sectores desfavorecidos; pregonamos que haya autogestión, que desarrolle pensamiento liberador, 
mientras nosotros trabajamos atomizados, de forma individualista y acrítica.

-F.G.R.: Ese es un punto clave. No hacer de nuestra reflexión comprometida con la ciencia y con el cambio, un recurso de politiquería para hacernos hegemónicos en la dimensión política tradicional.

-A.D.G.: Debemos ser capaces de llegar a acuerdos mínimos de cómo funcionar como gremio.

-F.G.R.: En eso estoy de acuerdo, hoy trabajamos en parcelas, líneas dispersas y habría que organizar un movimiento que implica seguridad, lealtad entre las personas. Si tú analizas los textos de nuestra época, reconocerás que allí había muchas situaciones recurrentes, había un movimiento que respiraba en la diversidad, porque nosotros nunca tuvimos posiciones semejantes unas de otras, muchas diferencias han permanecido hasta hoy, lo importante es ver cómo nos alimentábamos unos de otros.

-A.D.G.: En esa diversidad podemos demarcar, cómo, al hablar de psicologías sociales críticas, reconocemos actualmente, en América latina, las siguientes tendencias en pluralidad: la psicología de la liberación, la psicología comunitaria que pude contener rasgos de la psicología crítica o no, la psicología social latinoamericana crítica, la psicología política crítica, algunas de ellas enmarcadas en la psicología social marxista que nos llegó desde los desarrollos de Cuba y, por esta vía, o de forma directa de la denominada en ese momento, Unión Soviética. ¿Tiene aún vigencia esta psicología social marxista?

-F.G.R.: Pienso que hasta el propio Vigotsky intentó separar la psicología del atributo marxista, pues la cuestión no es definir una psicología marxista, sino reconocer cómo el Marxismo definió, influyó decisivamente en un nuevo tipo de psicología. Los psicólogos cubanos, hace tiempo, cuando empezamos a desarrollar los encuentros internacionales de reflexión con el psicoanálisis y su gama de densidades, reconocimos que hay una psicología social con base psicoanalítica, Pichon Rivière fue un psicólogo social crítico. No me gusta crear la crítica por tendencias, porque se escapan cosas.

El problema, al hablar de una psicología social marxista, está en que el Marxismo no es el fin o el tope de una producción crítica, hay autores no marxistas que han sido y que son críticos. Nosotros dejamos de llamarnos psicología marxista, para llamarnos psicología de orientación marxista, parece lo mismo, pero no lo es, porque el marxismo participa en el camino de esa psicología, ha sido una influencia importante, pero no la agota en sus diferentes posibilidades.

El Marxismo fue decisivo en la entrada de la cultura y lo social en la psicología, en la producción de lo individual desde una perspectiva diferente. Creo que es el gran aporte que hace Vigotsky. Porque el Marxismo se nutre de una dialéctica que el propio Edgar Morín, en su autobiografía denominada Mis demonios, reconoce lo ayuda para realizar todas las construcciones que en sus desdoblamientos son su base de la comprensión y propuesta de la teoría de la complejidad.

Pero hablar de una psicología social marxista nos puede traicionar, porque es como un acto de fe, es partidarizar: yo soy marxista. Hay que tener cuidado con las substancializaciones que viran en ejes conservadores. El Marxismo fue una doctrina de cambio importante que alimentó e integró a un pensamiento, la complejidad de dimensiones múltiples de lo social en un pensar dialéctico, con un modelo que integra la contradicción y el movimiento. Sin embargo, a veces, nos vemos con marxistas que detienen el movimiento y la contradicción, y que intentan postular el Marxismo como entidad y no como una forma de ver el mundo, ahí, me separo de eso.

-A.D.G.: Hay un texto de Thénon (1974) titulado Psicología dialéctica, pero esta no necesariamente implica psicología marxista.

-F.G.R.: iNo! La dialéctica es una herramienta que Marx pule y que se integra a la psicología de una manera más eficiente. El propio Jung habla del método dialéctico, sin una impronta del pen- 
samiento de Marx, pues no lo he visto citado en sus obras, pero lo hace con una apreciación sobre la sutileza que la dialéctica tenía para los procesos de construcción de la psicología.

-A.D.G.: Hay un texto de Petrovski (1986) sobre psicología del colectivo ies vigente aún esta denominación o fue algo que correspondió a una época de desarrollo de la psicología soviética?

-F.G.R.: Creo que fue la marca de una época y la inversión de la palabra colectivo, cuando lo que se pretendía con ese pretendido colectivismo era una subordinación del grupo a las voces hegemónicas. Uno de los errores de la visión soviética de lo social, fue reducir esto a lo colectivo. Vigotsky lo criticó en su momento, porque lo social son practicas simbólicas que están más allá del espacio colectivo y que tienen su génesis en la forma de organización e institucionalización de una vida social que trasciende mi espacio de acción con el otro, aunque esté atravesado por este. Lo colectivo me habla del grupo, del contacto con el otro y, claro, que esa es una dimensión social por excelencia, pero no se reduce a eso.

-A.D.G.: Habiendo múltiples posibilidades de seguir conversando sobre este tema, le quiero proponer una hipótesis -en dos vías- que estoy trabajando para saber su perspectiva. Primero: la psicología de la liberación es -o podría ser- una manera de expresión de la psicología política en América Latina. Segundo: la psicología de la liberación ayuda a integrar perspectivas de la psicología comunitaria en cuanto a sus principios de trabajo con la comunidad tales como: empoderamiento, emancipación, desarrollo de la comunidad; la psicología de la liberación también podría desarrollar principios que la sustentan en sí (Guzzo \& Lacerda, 2009), con el cruce de la psicología política, en términos de categorías como liberación, acceso al poder.

Se puede argumentar que esta hipótesis es un híbrido burdo, en cuanto se planea una relación entre la psicología de la liberación y la psicología política, pero cuando uno mira en detalle los discursos de la psicología de la liberación puede reconocer que esta es la forma más cercana de aproximarnos a la psicología política. Parece que ha tenido mayor forma de desarrollarse en la última década la psicología de la liberación -de hecho, acaba de realizarse el X Congreso Internacional de Psicología de la Liberación-que la psicología política en términos de la comunidad académica y de la difusión de su producción intelectual. ¿Por qué esa fuerza de la primera, respecto de la segunda, si no es porque ella en sí misma logra recoger a la psicología política?

-F.G.R.: Ese es un tema complicado que me gustaría pensar más. No creo que los congresos de psicología de la liberación hayan marcado un desarrollo de una psicología que se pueda denominar de la liberación. He participado de varios de estos encuentros y creo que son un glosario de trabajos disímiles, todos con buenas intenciones políticas, con orientación social, pero que no ha marcado un crecimiento teórico de esta perspectiva de la psicología. De todas formas, el esfuerzo de un grupo importante de personas, entre ellos J. M. Flores, la propia Maritza Montero e Ignacio Dobles, en la organización de esos congresos, ha sido una importante contribución. Creo que la psicología de la liberación quedó en Martín Baró muy bien estructurada, con muchas avenidas para ser construidas a partir de sus planteamientos. Pero, a Martín Baró lo mencionamos mucho y no lo hemos trabajado en profundidad, en los diálogos de las nuevas alternativas y núcleos generadores de pensamiento con lo que fue su ideario fundante.

Creo que pensar la liberación debe integrar dos tendencias que se desarrollaron con independencia en América Latina: Paulo Freire y Martín Baró, a quienes les veo muchos puntos de contacto: ambos refieren la acción de liberación al trabajo esencial con grupos sociales, donde el concepto de liberación lo ven como producciones de alternativas al interior de la acción con la comunidad; sin embargo, ninguno de los dos tiene un proyecto político de toma del poder. Me acuerdo de una frase clave de Martín Baró: "entre la revolución y el cambio hay muchos momentos que no se acercan a la revolución"; ni Martín Baró, ni Freire tenían un discurso político prefabricado. Tenían una concepción de liberación 
que estaba orientada a intentar crear nuevos sujetos sociales en los sectores totalmente enajenados, fuera del poder latinoamericano.

En un libro que coordina Bernardo Jiménez, escribí unas críticas a la psicología de la liberación en el sentido de no substancializar este concepto (González Rey, 2008). Aunque el tiempo nos va flexibilizando para ver las limitaciones de nuestras ideas, pero también sus posibilidades. Hoy me siento como un interlocutor con nuevas ideas que me surgen, pero de eso no tengo nada escrito.

Creo que es importante el concepto de liberación en tanto demarca ruptura, no es un fin para poder decir: llegamos a la libertad. Esto sería substancializar un estado con una dimensión política, que es lo que a mí siempre me preocupa. Porque he visto que los libertadores se vuelven totalitarios. Es negar la dialéctica de la condición humana; hoy puedo ser un libertador del discurso académico y la adulación, el poder, el ir ocupando un lugar cada vez más central que me va referenciando, me llevan a ser un tipo vanidoso, acrítico y comienzo a substancializar mis aportes con un fin, y iahí se acabó la liberación!

Sin embargo, no fue esto lo que ocurrió ni con Martín Baró, ni con Paulo Freire. Este tiene una noción explícita del sujeto, ifantástica! En Pedagogía del oprimido. Por su parte Martín Baró, quien estaba permanentemente creando nuevos ejes de expresión de su pensamiento, pero él no titulaba la liberación en las tendencias políticas tradicionales y era sumamente cauto para vincularse con las definiciones tradicionales dominantes en el campo político. En aquel momento, é apoyaba el movimiento guerrillero salvadoreño, porque era la expresión del progreso frente a las fuerzas conservadoras, no porque fuera un movimiento que devendría en una alternativa última. Él era cuidadoso y veía la resistencia como producción social y como expresión de la integración y activación de fuerzas de la sociedad que no tenían participación; Freire, igual. Los dos tienen una capacidad extraordinaria, no dogmática. Sus obras van progresando en diversidad.

Martín Baró fue muy abierto a la reflexión, nunca fue un idealizador de nada, fue bastante crítico. Es importante separar el concepto de liberación de fórmulas políticas a priori o dominantes en el escenario actual, que a veces se quieren relacionar con la liberación. Tenemos que tener cuidado con las retóricas políticas que se autodenominan progresistas. Me inclino a pensar que la política es progresista siempre en contexto, no por filiaciones partidarias definitivas. Los partidos, que en un momento son progresistas, pueden pasar a ser conservadores.

-A.D.G.: Insisto con la pregunta: ila psicología de la liberación, es expresión de la psicología política?

-F.G.R.: Creo que es psicología política. No una expresión. Es psicología política. iUna psicología eminentemente política! Por la vinculación de nuevos actores que no están en el discurso político tradicional, pero que a la vez entran como actores de una sociedad con programas y objetivos que los convierte en fuerzas políticas. Porque hay que diferenciar la politiquería, o la política operacional como tú la llamaste, de la política como sentido de acción en los marcos sociales.

\section{II.- ¿Subjetividad política? ¿Subjetividad social?}

-A.D.G.: Fernando, en el contexto que usted demarca de producción y huella teórica de la psicología social en Latinoamérica, me parece que, en el caso de Colombia, hay un núcleo emergente, muy marginal, desde el cual estamos trabajando el tema de la subjetividad. Pero, seguramente por el contexto en el cual estamos viviendo, por las condiciones sociopolíticas específicas de nuestro país, hemos asumido el abordaje de la subjetividad política (Calderón, 2009; Díaz, 2007; Martínez, 2009; Muñoz, 2007) de la cual usted toma distancia, en cuanto, como lo ha dicho previamente, se ubica en la subjetividad social y, como quedó consignado en otras entrevistas (Díaz, 2005, 2006), para usted la subjetividad política está inmersa en la subjetividad social.

Sin embargo, creo que es posible llegar a hablar de subjetividad política como condición del sujeto que se expresa en cuanto sujeto político, por vía 
del ejercicio de la ciudadanía. Ese sujeto tiene particularidades en términos de la subjetividad que se pueden desdoblar en múltiples entradas, una de ellas la política (Alvarado, Ospina \& Muñoz, 2008; Sánchez, Hensel, Zuleta \& Pedraza, 2010). Aquí me apoyo e intento ayudar en el desarrollo de dos categorías que usted ha trabajado, como son las de sentido y sentido subjetivo, para plantear cómo el sujeto político expresa su subjetividad política mediante sentidos subjetivos que son específicos de lo político.

En mis reflexiones, me interesa relacionar los sentidos subjetivos con lo político, para hacer un diálogo interdisciplinar entre la psicología -con la psicología social, en particular mediante la categoría de subjetividad- y la ciencia política que ha sido una ciencia más instrumental y positivista, para tratar de reconocer cómo allí, desde la ciencia política, se puede llegar a trabajar no solo elementos operativos de la política como el voto, niveles de favorabilidad de candidatos, sino, cómo incluso en esas acciones de elección el sujeto debe constituirse como sujeto político y hacer acciones de reflexividad, que le implican tomar posturas respecto de lo público, es decir, de lo que nos es común, allí se constituiría lo político. Pero cuando él toma postura de lo político, va asumiéndose como sujeto político. En cuanto estos planteamientos tienen cierta coherencia, algunos niveles de desarrollo teórico e investigativo son un aporte a esa psicología social de Latinoamérica. ¿Cómo ve usted estos argumentos desde una mirada más amplia y externa?

-F.G.R.: Creo que son ideas muy razonables. Además, siempre he estado en contra de la substancialización de las categorías, creo que estas hay que pluralizarlas. La subjetividad política es una producción de la subjetividad social, que tiene especificaciones importantes que vale la pena estudiar y que la convierten en un campo de conocimiento. Una preocupación que me asalta es que cuando se habla de subjetividad política $-y$ se tiene en cuenta mi propuesta sobre subjetividad-nunca la podemos referir a un contenido aislado de otros.

Cuando hablamos de subjetividad política, o en los viejos términos, la motivación política, debemos tener en cuenta que -lo veo más claro, sobre todo ahora, después de vivir durante tantos años en un país latinoamericano con esa policromía que se da en lo político, pero que va creando formas de subjetivación dominante que hacen muy difícil las rupturas y las alternativas- los procesos públicos que están dados en la organización política, generan formas de subjetivación que hay que considerarlas en el análisis político para evitar la política dessubjetivada que tú has mencionado y que ha acompañado a nuestros países. Sin embargo, el énfasis que quiero hacer, es que, en esa subjetividad política entran otros procesos que se configuran en sentidos subjetivos con connotación política (en forma de sentidos subjetivos que no necesariamente son del orden político) y que terminan configurando la manera en que se organiza el orden político hoy. Por ejemplo, cuando discuto con los brasileños, argumento cómo nosotros tenemos en América Latina una crítica monolítica poco diferenciada del capitalismo, el gran muñeco de los golpes. Sin embargo, el capitalismo brasileño, como el de cualquier país, se alimenta de aspectos históricos y contextuales que definen su organización única en Brasil, como el coronelismo. Los coroneles hegemonizaron todo el nordeste brasileño y desarrollaron una política con consecuencias subjetivas extremadamente conservadoras como el nepotismo, la impunidad y la falta de participación, que están muy presentes en la política brasileña hoy, en los intocables de la política. El racismo de nuestros países viene del esclavismo y toma formas sutiles, que entran en los sistemas de relaciones humanos de los que emergen sentidos subjetivos que ganan dimensiones políticas. La cuestión de la raza, como elemento que toma forma política en determinadas configuraciones, se articula con otros procesos de organización de la subjetividad social y se alimenta de muchísimas cosas: de las propias motivaciones, de la realización de los actores políticos, de los sujetos individuales que viran en sujetos de la acción política. Nunca el sujeto político es tan específico como nosotros nos hemos esforzado en verlo.

El sujeto político trae motivaciones de muchas índoles, entre ellas las de dominancia y las de hegemonía. Nosotros hemos visto que, en América 
Latina, procesos revolucionarios importantes, incluyendo el cubano, han tenido perversiones notorias por perpetuidades en el poder que son difíciles de pensarlas como progresistas. Estos son temas complicados. Ya Carlos Fuentes, en su fantástico libro La muerte de Artemio Cruz, nos recuerda que "Las revoluciones las hacen los hombres de carne y hueso y no los santos, y todas acaban por crear una nueva casta privilegiada". Los hombres pasan a ser mitificados como dioses y, con eso, llega a su fin cualquier intención progresista que haya animado su acción anterior.

Lo que no me gusta es separar una subjetividad política de una subjetividad que tiene muchas entradas, en el sentido de sus configuraciones, sin embargo, creo que la especificidad del tema de la subjetividad política permite generar problemas concretos al estudio de la política, que sin el término quizá no existirían. Hoy, soy mucho más afín a este tema, que antes. Lo que he dicho no niega la posibilidad de trabajar la subjetividad política, pero da una alerta de que no todo lo que está en ella es de orden político. Los sentidos subjetivos nunca tienen límite, dadas las múltiples configuraciones de las que provienen y asumen. Precisamente, ahí veo el valor heurístico de la categoría configuración subjetiva: en su carácter flexible y maleable, para integrar una pluralidad de sentidos subjetivos que expresan cosas diferentes de la vida social del hombre, tanto del sujeto actuante como de la movilidad de los espacios sociales y su interpenetración.

-A.D.G.: Fernando, en eso coincidimos y podemos seguir complementando en el sentido que pensar la subjetividad política implica dos desplazamientos, uno: un desplazamiento para repensar la categoría de la política (Díaz, 2007b, 2008), para que esta no sea solo la parte procedimental para concretar formas de gobierno, sino que sea asumida incluso como el ejercicio "del poder en los ámbitos de la vida cotidiana" y, segundo, relacionarla con la categoría de lo político (Díaz, 2003) que asume un potencial distinto en cuanto ya no es lo procedimental, sino lo procesual. Lo político es lo que podemos llegar a trabajar dentro de los procesos de subjetivación política, estos no se hacen sobre los procesos de la política, sino de lo político.

Sobre este aspecto, poco o nada se ha trabajado desde los ámbitos de la psicología social y de la psicología política, donde predomina, todavía, la reflexión sobre categorías tradicionales como la intención de voto, las actitudes frente a procesos de la política, el liderazgos político, no reconociendo procesos emergentes, que, como el de la subjetividad, entran nuevamente en circulación, para la discusión e investigación científica, adjetivados como subjetividad política.

Asumir este tema como parte de la agenda teórica de la psicología social, conlleva dos consecuencias: repensar alternativas de investigación cualitativas que permitan capturar las formas como se expresa la subjetividad política y reconocer que esta no es una categoría fundante, única y aislada, sino que tiene que ser integrada, como por ejemplo, con la producción teórica existente sobre lo político desde donde se reconoce que este tiene una dimensión de emoción. Es decir, se deben hacer nuevos entramados conceptuales para pensar la subjetividad política -aun, siguiendo su propuesta- como un desdoblamiento de la subjetividad social en su particularidad. Teniendo claro que no pretendemos hablar de un sujeto político aislado, separado de su subjetividad social, sino que lo asumimos como un sujeto que se constituye, precisamente, en la relación y entre la relación social.

-F.G.R.: Ese es un tema que tiene mucho horizonte para ser trabajado y una cantidad de formas que están presentes en lo político, y que tenemos que descubrir en lo cotidiano, porque se nos oculta a la apariencia.

Me gusta la línea de conclusión que has expresado. Solo quería decirte que tenemos que tener cuidado con la categoría de poder, pues se ha reificado mucho, se volvió una moda que la ha reificado desde el planteamiento de Foucault, sin duda, un pensador crítico. Pero las prácticas de poder también están dadas en formas de organización social que pasan por lo económico, por las formas de distribución de la propia organización de la vida social, 
por formas de organización de lo cotidiano, por muchas formas que no son solo prácticas de poder.

El poder también está establecido y se expresa sobre realidades que ejercen una tremenda presión en los protagonistas, pues son parte de realidades históricas y sociales muy complejas, que también se están organizando en nuestras configuraciones subjetivas a través de las cuales el poder se ejerce.

El poder como práctica que toma forma en todas las expresiones de la vida, es un poder que tiene rutas dadas por formas de organización social. Creo que Marx lo planteó -y todavía tiene una pertinencia importante-, cuando dijo como el acto humano está organizado en su dimensión subjetiva, por desdoblamientos de prácticas de formas de vida que tienen estructuras muy complejas en lo social, que están ocultas.

-A.D.G.: Sin embargo, se debe reconocer que hablar de la política, de lo político, implica relacionarlo con el poder.

-F.G.R.: Siempre, lo político es una forma de ejercicio del poder.

-A.D.G.: Aunque con los matices particulares que en la actualidad puede tener.

-F.G.R.: Sí, porque, por ejemplo, la política está representando formas de organización que a veces no se dan explícitas en el poder. Sin embargo, están por detrás de todas las formas de poder.

\section{Referencias}

Alvarado, S. V, Ospina, H. F. Botero, P. \& Muñoz, G. (2008). Las tramas de la subjetividad política y los desafíos a la formación ciudadana en jóvenes. Revista Argentina de Sociología, 6(11), 19-43.

Botero, P., Cardona, M. \& Loaiza, J. (2007). Subjetividad y política: una perspectiva performativa. Revista de Ciencias Humanas, 12, 137-155.

Christlieb, P. (2004). El espíritu de la calle. Psicología política de la cultura cotidiana. Barcelona: Anthropos.

Calderón, A. (2009). Psicología política y subjetividad. Hacia la construcción de una mirada estético polí- tica del ejercicio político. Perspectivas en Psicología, 12, 43-50.

Correa, A. (Comp.). (2003). Notas para una psicología social como crítica a la vida cotidiana. Córdoba: Editorial Brujas.

Díaz, A. (2003). Una discreta diferencia entre la política y lo político y su incidencia sobre la educación en cuanto socialización política. Reflexión Política, 5(9), 49-60.

Díaz, A. (2005). Subjetividad: una perspectiva históricocultural. Conversación con el psicólogo cubano Fernando González Rey. Universitas Psychologica, 4(3), 373-383.

Díaz, A. (2006). Subjetividad y subjetividad política [Entrevista con el psicólogo cubano Fernando González Rey]. Revista Colombiana de Educación, 50, 170-182.

Díaz, A. (2007a). Subjetividad e institucionalidad educativa. Revista de Ciencias Humanas, 12, 91-101.

Díaz, A. (2007b). Entrevista a Cristina Sánchez Muñoz. Discusiones Filosóficas, 8(11), 299-313.

Díaz, A. (2008). Vigencia y pertinencia del pensamiento de Hannah Arendt: sus aportes sobre el totalitarismo. Revista de Estudios Sociales, 31, 164-171.

Díaz, A. (2009a). La producción de conocimiento sobre subjetividad política desde los jóvenes: aportes conceptuales y metodológicos. Cuadernos del Cendes, 26(70), 127-140.

Díaz, A. (2009b). Historia reciente (2000-2009) de la Psicología Social en Colombia: una mirada desde la Red Colombiana de Psicología Social Crítica. Psicolatina, 17. Recuperado el 7 de enero, 2011, de http://www.psicolatina.org/17/colombia.html

Fuentes, C. (2008). La muerte de Artemio Cruz. Bogotá: Punto de Lectura.

Jiménez, B. (1994). Cambios sociopolíticos y desarrollos históricos en psicología. En M. Montero (Coord.), Construcción y crítica de la psicología social (pp. 1126). Barcelona: Anthropos.

González Rey, F. \& Valdés, H. (1994). Psicología humanista. Actualidad y desarrollo. La Habana: Editorial de Ciencias Sociales.

González Rey, F. (1997). Epistemología cualitativa y subjetividad. La Habana: Editorial Pueblo y Educación.

González Rey, F. (1999). La investigación cualitativa en psicología. Rumbos y desafíos. Sao Paulo: Educ. 
González Rey, F. (2002). Sujeto y subjetividad. Una aproximación histórico-cultural. México: Thomson.

González Rey, F. (2004). O social na psicología e a psicología social. A emergencia do sujeito. Río de Janeiro: Editora Vozes.

González Rey, F. (2007). Investigación cualitativa y subjetividad. Los procesos de construcción de la información. México: MacGraw-Hill.

González Rey, F. (2008). Subjetividad y psicología crítica: implicaciones epistemológicas y metodológicas. En B. Jiménez (Comp.), Subjetividad, participación e intervención comunitaria. Una visión crítica desde América Latina (pp. 40-45). Buenos Aires: Paidós.

González Rey, F. (2010). El pensamiento de Vygotsky: contradicciones, desdoblamientos y desarrollo. México. Trillas.

Guzzo, R. \& Lacerda, F. (Orgs.). (2009). Psicología social para a América Latina. O resgate da psicología da liberacao. Campinas: Editora Alinea.

Martín Baró, I. (1988). Acción e ideología. Psicología social desde Centroamérica. El Salvador: UCA Editores.

Martín Baró, I. (1989). Sistema, grupo y poder. Psicología social desde Centroamérica II. El salvador: UCA Editores.

Martín Baró, I. (1990). Psicología social de la guerra. El salvador: UCA Editores.

Martínez, J. (2009). Arqueología y genealogía para una nueva subjetividad: la ética del cuidado de sí. En J. Martínez \& F. Neira (Eds.), Miradas sobre la subjetividad. Bogotá: Universidad de la Salle.

Montero, M. (Coord.). (2002). Psicología comunitaria. Teoría, método y experiencia. México: Universidad de Guadalajara.

Montero, M. (2003). Teoría y práctica de la psicología comunitaria. La tensión entre comunidad y sociedad. Buenos Aires: Paidós.
Montero, M. (2004). Introducción a la psicología comunitaria. Desarrollo, concepto y procesos. Buenos Aires: Paidós.

Montero, M. (2010). Crítica, autocrítica y construcción de teoría en la psicología social latinoamericana. Revista Colombiana de Psicología, 19(2), 177-191.

Moscovici, S. (1993). La era de las multitudes. Un tratado histórico de psicología de las masas. México: Fondo de Cultura Económica.

Munné, F. (1982). Psicologías sociales marginadas. La línea de Marx en la psicología social. Barcelona: Editorial Hispano Europea.

Muñoz, G. (2007). Identidades o subjetividades en construcción. Revista de Ciencias Humanas, 12, 69-91.

Obando, L. (Comp.). (2010). Psicología social crítica: aportes y aplicaciones sobre el lenguaje, ciudadanía, convivencia, espacio público, género y subjetividad. Cali, Colombia: Universidad del Valle.

Petrovski, A. (1986). Teoría psicológica del colectivo. La Habana: Editorial Ciencias Sociales.

León, J., Barriga, S. \& Gómez, T. (1998). Concepto y características metodológicas de la psicología social. En J. León, S. Barriga, T. Gómez, B. González, S. Medina \& F. Cantero (Coords.), Psicologia social: orientaciones teóricas y ejercicios prácticos (pp. 3-24). Madrid: MacGraw-Hill.

Saforcada, E. \& Castella, J. (Comps.). (2008). Enfoques conceptuales y técnicos en psicología comunitaria. Buenos Aires: Paidós.

Sánchez, A., Hensel, F., Zuleta, M. \& Pedraza, Z. (Comps.). (2010). Actualidad del sujeto. Conceptualizaciones, genealogías y prácticas. Bogotá: Universidad Central/Universidad de los Andes/Universidad del Rosario. 